\title{
A helium P-Cygni profile in RR Lyrae stars?^
}

\author{
D. Gillet ${ }^{1}$, F. L. Sefyani ${ }^{2}$, A. Benhida ${ }^{2}$, N. Fabas ${ }^{3}$, P. Mathias ${ }^{4,5}$, Z. Benkhaldoun ${ }^{2}$, and A. Daassou ${ }^{2}$ \\ ${ }^{1}$ Observatoire de Haute-Provence - CNRS/PYTHEAS/Université d'Aix-Marseille, 04870 Saint-Michel l'Observatoire, France \\ e-mail: denis.gillet@osupytheas.fr \\ 2 Observatoire d'Oukaïmeden, Faculté des Sciences et Techniques, Département de Physique, LPHEA, Marrakech, Morocco \\ e-mail: [sefyani; a.benhida; zouhair; daassou]@uca.ma \\ 3 Kiepenheuer-Institut fur Sonnenphysik, Schöneckstr.6, 79104 Freiburg, Germany \\ e-mail: fabas@kis.uni-freiburg.de \\ ${ }^{4}$ Université de Toulouse, UPS-OMP, Institut de Recherche en Astrophysique et Planétologie, 31400 Toulouse, France \\ 5 CNRS, UMR 5277, Institut de Recherche en Astrophysique et Planétologie, 14 avenue Édouard Belin, 31400 Toulouse, France
}

Received 1 September 2015 / Accepted 26 November 2015

\begin{abstract}
Context. Until 2006, helium emission lines had never been observed in RR Lyrae stars. For the first time, a pre-maximum helium emission in $11 \mathrm{RRab}$ stars was observed during rising light (around the pulsation phase 0.92) and the reappearance of helium emission near maximum light (phase 0.0) in one RRab star: RV Oct. This post-maximum emission has been only observed in the He I $\lambda 5875.66$ (D3) line. Its intensity is very weak, and its profile mimics a P-Cygni profile with the emission peak centered at the laboratory wavelength. The physical explanation for this unexpected line profile has not been proposed yet.

Aims. Using new observations of RR Lyr, we investigate the physical origin of the presence of a P-Cygni profile in the He I $\lambda 5875.66$ (D3) line.

Methods. High-resolution spectra of RR Lyr, collected with a spectrograph eShel/C14 at the Oukaïmeden Observatory (Morocco) in 2013, were analyzed to understand the origin of the observed P-Cygni profile at D3.

Results. When the shock intensity is moderate, helium emission cannot be produced in the shock wake, and consequently, the two consecutive helium emissions (pre- and post-maximum light emissions) are not observed. This is the most frequent case. When the shock intensity becomes high enough, a pre-maximum He I emission first occurs, which can be followed by the appearance of a P-Cygni profile if the shock intensity is still strong in the high atmosphere. The observation of a P-Cygni profile means that the shock wave is already detached from the photosphere. It is shown that the shock strongly first decelerates between the pulsation phases 0.90 and 1.04 from $130 \mathrm{~km} \mathrm{~s}^{-1}$ to $60 \mathrm{~km} \mathrm{~s}^{-1}$, probably before accelerating again to $80 \mathrm{~km} \mathrm{~s}^{-1}$ near phase 1.30 .

Conclusions. The presence of the P-Cygni profile seems to be a natural consequence of the large extension of the expanding atmosphere, which is induced by strong (radiative) shock waves propagating toward the high atmosphere. This kind of P-Cygni profile has already been observed in the $\mathrm{H} \alpha$ line of some RR Lyrae stars and long-period Cepheids.
\end{abstract}

Key words. stars: atmospheres - stars: variables: RR Lyrae - stars: individual: RR Lyrae - radiation: dynamics - shock waves

\section{Introduction}

There are three emission episodes within the $\mathrm{H} \alpha$ profile during one pulsation cycle in RR Lyrae stars. The first and strong emission that occurs near the pulsation phase 0.92 is called the "first apparition" by Preston (2011). A secondary $\mathrm{H} \alpha$ emission (the "second apparition") has been discovered by Gillet \& Crowe (1988) during the pre-minimum brightening near the pulsation phase $\varphi=0.72$. It is produced when outer atmospheric layers in ballistic infall collide with inner, more slowly photospheric layers. A third weak emission (the "third apparition"), occurring at phase 0.3 has been recently observed in the red absorption wing of the $\mathrm{H} \alpha$ profile by Preston (2011). Gillet \& Fokin (2014) propose that the "third apparition" (a P-Cygni profile) is produced by the main shock when it is far enough from the photosphere.

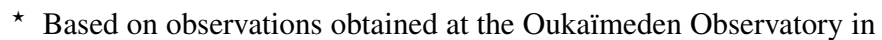
the High Atlas mountains, $78 \mathrm{~km}$ south of Marrakech and operated by the Faculté des Sciences Semlalia, Département de Physique, LPHEA, Marrakech, Morocco.
Thus, the emission is the natural consequence of the large extension of the expanding atmosphere.

Whitney (1956) first discussed the presence of helium emission as an extension to hydrogen emission. Since hydrogen emission is thought to be due to radiative cooling of the gas compressed by the shock propagating through the atmosphere in each pulsation cycle, Whitney (1956) found that a shock front with a velocity higher than $80 \mathrm{~km} \mathrm{~s}^{-1}$ has enough energy to completely ionize $\mathrm{H}, \mathrm{He}$ I, and a small fraction of He II. Wallerstein (1959) estimated that it is quite possible to observe helium emission lines; however, no one observed any helium emission in RRab stars during the next half-century.

Pre-maximum helium emissions in RR Lyrae stars were unexpectedly found by Preston (2009) in a sampling of 11 observed RRab stars, among which eight are Blazhko stars. The strongest He I emission (D3 line at $\lambda 5875.66$ and the weaker emission at 26678.16$)$ were observed in all observed stars during rising light. These emissions occur when hydrogen also appears in emission around the pulsation phase 0.92 . Then, a line-doubling 
absorption profile is observed, as for hydrogen. In addition, a very weak He II emission at $\lambda 4685.68$ was also detected at the same phase, but only in three Blazhko stars.

Helium emissions are expected to be formed in the deexcitation region of the shock wake. However, to produce the observed helium emissions, it is necessary that the temperature and the density in the de-excitation region of the wake are high enough. As shown first by Fokin \& Gillet (1997) for RRab, the final amplitude of the main shock can be as high as $120-170 \mathrm{~km} \mathrm{~s}^{-1}$, i.e., a high Mach number (between 15 and 20). Within this range of Mach numbers, Fadeyev \& Gillet (2001) have shown that the temperature and density in the deexcitation region should be high enough to produce He I and He II emissions for highest shock velocities. A temperature of $25000-50000 \mathrm{~K}$ and a density $50-100$ times higher than in the unperturbed gas are expected. Thus, the presence of helium emissions strongly depends on the intensity of the shock wave. In particular, to observe He II, the temperature behind the shock must be high enough to ionize He I. Consequently, the detection of $\mathrm{He} \mathrm{I}$ and $\mathrm{He}$ II emissions provide fundamental informations about the shock intensity (Gillet \& Fokin 2014). This is why Gillet et al. (2013) define three hydrodynamic regimes: (a) the supersonic regime in which only hydrogen emissions are visible; (b) the weak hypersonic regime where the He I emission lines are also observed; and (c) the strong hypersonic regime with the additional observation of He II emission lines. Up to now, the last regime has only been observed in Blazhko stars.

For the first time, Preston (2009) also observed a postmaximum helium emission in the D3 line in one RRab Blazhko star: RV Oct during about $5 \%$ of the pulsation cycle around the phase $\varphi=0.30$. The intensity of this emission is weak (about $2 \%$ of the continuum) and mimics a P-Cygni profile with emission centered on the stellar rest frame velocity. The physical explanation of the reappearance of helium emission near maximum light is not known yet.

The third emission occurring simultaneously within the $\mathrm{H} \alpha$ profile (the "third apparition"), which has been first observed by Preston (2011), is difficult to detect because of both its weak intensity and its occurrence in the red absorption wing of the large photospheric absorption. This small emission shoulder is present near phase $\varphi=0.3$, i.e., after maximum light. Consequently, it is hard to know its exact phase of appearance. Gillet \& Fokin (2014) were the first to suggest a physical mechanism to explain this weak redshifted emission. They assume that the shock wave is already sufficiently detached from the photosphere when the redshifted $\mathrm{H} \alpha$ line appears. Thus, if the emitting gas is not yet completely optically thick, a P Cygni profile is formed.

It should be noticed that the phase in which the so-called "third apparition" occurs, is near maximum photospheric radius. Most of the metallic lines are formed near the photosphere, while the hydrogen and helium line cores are formed above the photosphere. Thus, a moderate acceleration of the upper atmosphere is still possible when the photospheric radius is at its maximum (near 0.3). This phase is often adopted as one where the metallic line profile are "the most stable" for determining abundances (see, e.g., Kolenberg et al. 2011). Thus, the layers in which the metal lines are formed seem weakly affected by phenomena occurring in the upper atmosphere such as the third apparition. As a consequence, the use of metallic lines in the phase of maximum photospheric radius may still be the best option when determining abundances.

In this paper, we present the observation of the D3 helium P-Cygni profile in another RRab, Blazhko star for the first time,
RR Lyr itself. In Sect. 2 we describe observational and data reduction processes. The detection of very weak helium P-Cygni profile is discussed in Sect. 3. The physical origin of the helium P-Cygni profile and its connection with the third $\mathrm{H} \alpha$ emission are discussed in Sect. 4. Finally, some concluding remarks are given in Sect. 5.

\section{Observations and data analysis}

On October 12, 2013, we used an optical fiber-fed echelle spectrograph eShel of the Shelyak Instruments company (Thizy \& Cochard 2010). The eShel system includes a F/6 Fiber Injection and Guiding Unit, a $50 \mu \mathrm{m}$ optical fiber, a ThAr Calibration Unit, and an echelle spectrograph (125 mm F/5 collimator, R2 echelle grating, cross-dispersing prism, $85 \mathrm{~mm} \mathrm{~F} / 1.8$ objective). The spectrograph was installed on a C14 Celestron telescope at the Oukaïmeden observatory situated at an altitude of $2700 \mathrm{~m}$ in the High Atlas mountains, $78 \mathrm{~km}$ south of Marrakech. The pixel size was $6.8 \mu \mathrm{m}$, and the spectral dispersion was $16 \AA / \mathrm{mm}$ or $0.1 \AA / p x$.

We obtained 39 consecutive spectra over a large part of the visible domain (from 4100 to $7200 \AA$ ), between orders 32 and 52 and with a resolution power of about 12000 . With an exposure time of $300 \mathrm{~s}$, the signal-to-noise ratio $(\mathrm{S} / \mathrm{N})$ is about 30 . The adopted temporal resolution $\Delta t / P=0.6 \%$ is low enough to ensure proper monitoring of the line profile evolution induced by nonlinear nonadiabatic pulsations occurring in the atmosphere. The data were reduced with the free and open source astronomy software AudeLA ${ }^{1}$. This package performs classical operations, such as bias subtraction, flat-fielding, masking of bad pixels, wavelength calibration, and spectrum extraction, including extraction of the spectroscopic information. The wavelengths are given in the rest frame of RR Lyr considering a star velocity $V_{*}$ with respect to the solar system barycenter of $-73.5 \mathrm{~km} \mathrm{~s}^{-1}$ (Chadid \& Gillet 1996).

Spectra were filtered with a Gaussian filter with a standard deviation of the distribution equal to 0.6 pixel. Knowing that the resolution of the spectrograph is around 12000 with a spectral dispersion of $0.1 \AA / \mathrm{px}$, this leads to a resolution of about five pixels for the D3 line, so this filter keeps the spectral resolution of our measurements.

The phases of our 39 spectra have been computed using following ephemeris, as indicated in the GEOS RR Lyr database ${ }^{2}$ :

$\operatorname{HJD}($ max light $)=2456578.450+0.56683561 E$.

Hereafter the pulsation phase is denoted by $\varphi$. With a $13.60 \mathrm{~h}$ pulsation period, our observations cover $25 \%$ of the pulsation period from phases $\varphi=0.853$ to 1.098 . This corresponds to an interval of $3^{\mathrm{h}} 20^{\mathrm{min}}$ from UT $20^{\mathrm{h}} 48 \mathrm{~min}$ to UT $00^{\mathrm{h}} 08^{\mathrm{min}}$.

Determining the Blazhko phase is more problematic because the Blazhko period shows apparent erratic changes (Le Borgne et al. 2014). For better accuracy, it is necessary to use a date of maximum light amplitude that is as close as possible to observations and a valid value for the period. To obtain an estimate of the Blazhko ephemeris, we used times of the light maximum for RR Lyr collected by the GEOS RRLyr web database around our observation date (October 12, 2013). Unfortunately, between October and December 2013, the number of O-C determinations is small because of bad weather. The maximum Blazhko amplitude (by definition phase $\psi=0.0$ ) occurred October 7, 2013 with

\footnotetext{
1 http://www . audela.org/

2 The GEOS database is freely accessible on the internet at http:// rr-lyr.irap . omp.eu/.
} 
D. Gillet et al.: A P-Cygni helium line profile in RR Lyrae stars

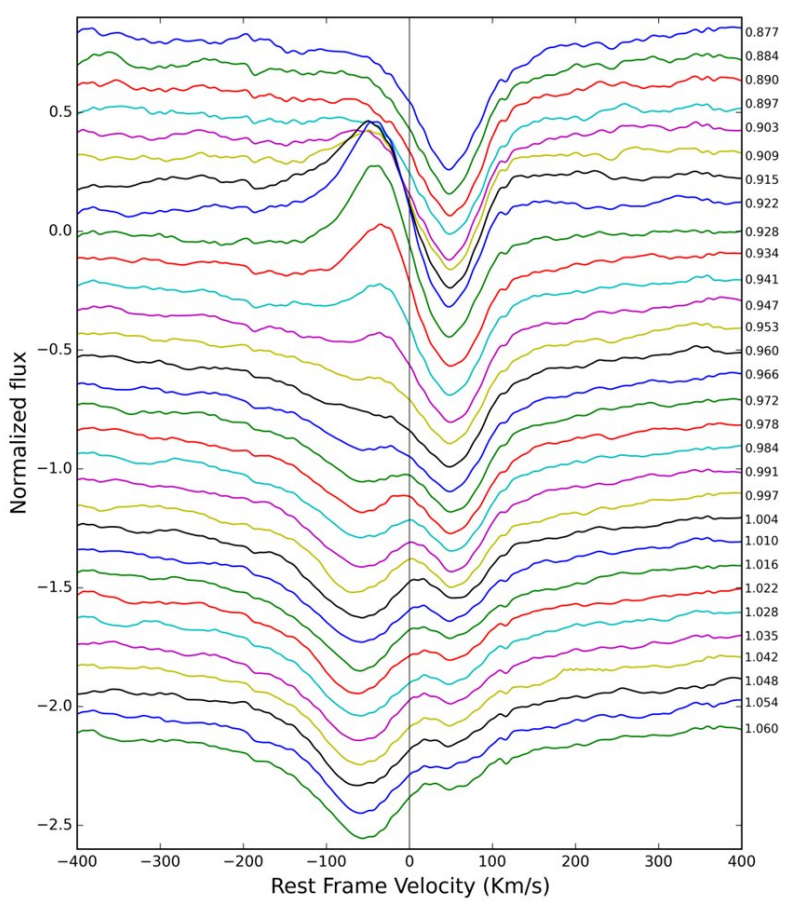

Fig. 1. Evolution of the $\mathrm{H} \alpha$ line profile of RR Lyr. The pulsation phases are indicated on the right side. The vertical line represents the zero velocity in the stellar rest frame.The profiles are arbitrarily shifted in flux.

a three-day uncertainty. Finally, we used the following Blazhko ephemeris:

$$
\operatorname{HJD}(\text { max. light ampl. })=2456572.5+39.0 E .
$$

It thus seems clear that our observations occurred just after a Blazhko maximum around $\psi=0.13 \pm 0.08$, i.e., just after the time when the strongest shocks occur. The error is estimated from the redistribution of $\mathrm{O}-\mathrm{C}$ points around the expected Blazhko maximum.

\section{The D3 helium P-Cygni profile in RR Lyr}

Figures 1 and 2 show the variation in the $\mathrm{H} \alpha$ line profiles (1Dand 2D-diagrams) around luminosity maximum. The blue emission shoulder, called the "first apparition" by Preston (2011), reaches its maximum intensity at $\varphi=0.922$. It is visible until $\varphi=0.941$ when its intensity is just below the continuum, and it is followed by the subsequent double absorption lines. As already discussed by Gillet et al. (2013), the profile variations of the double absorption follow the so-called Schwarzschild's mechanism closely (1952). This characteristic absorption line doubling is the consequence of the propagation of a shock wave throughout the atmospheric layers. The shock is a progressive wave producing a phase lag from the bottom to the top of the reversing layer. Our observations of the $\mathrm{H} \alpha$ line profile on October 12, 2013 are quite similar to those obtained at the CFHT (Mauna Kea, Hawaii) on July 4, 2011 with the ESPaDOnS spectropolarimeter (Gillet et al. 2013) and that correspond to similar pulsation (around light maximum) and phases $(\psi \sim 0.1)$ phases. The comparison of their two-dimensional map of the $\mathrm{H} \alpha$ profile (Fig. 1) with our Fig. 1b is quite demonstrative.

Just after the "first apparition" of the $\mathrm{H} \alpha$ emission shoulder (at $\varphi=0.903$ ), a sudden onset of the He I $\lambda 5875.66$ (D3) emission appears at $\varphi=0.909$ (Figs. 3 and 4). At this phase, it

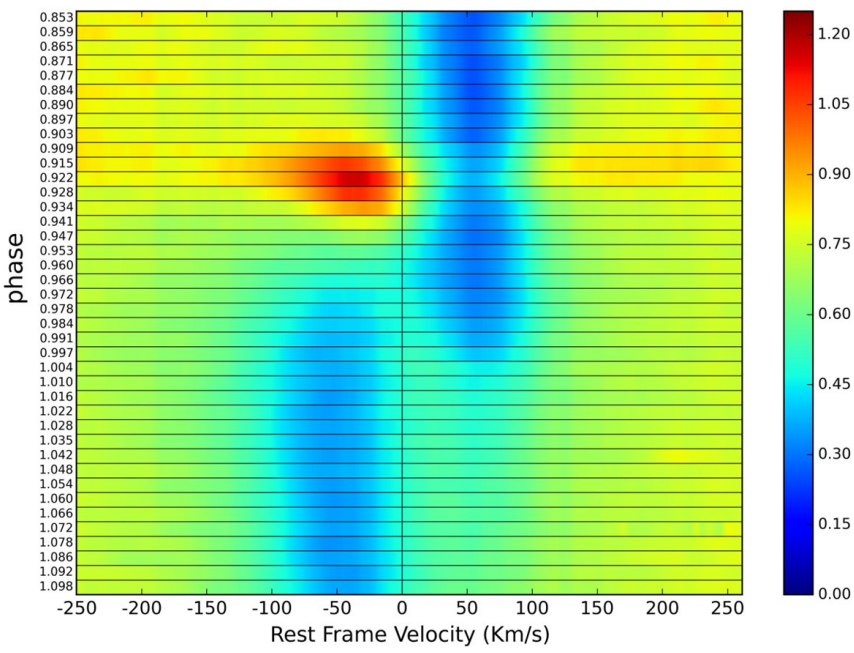

Fig. 2. Time series of $\mathrm{H} \alpha$ line of RR Lyr, ordered by increasing phase from top to bottom. Spectra are interpolated to provide a 2-dimensional map of the $\mathrm{H} \alpha$ profile in the $[-250,250] \mathrm{km} \mathrm{s}^{-1}$ velocity range. Velocities are given in the stellar rest frame with positive velocities corresponding to inward motion (toward the photosphere). The pulsation phase is given on the left side. On the right side, the color bar representing the flux is from 0.0 to 1.2 .

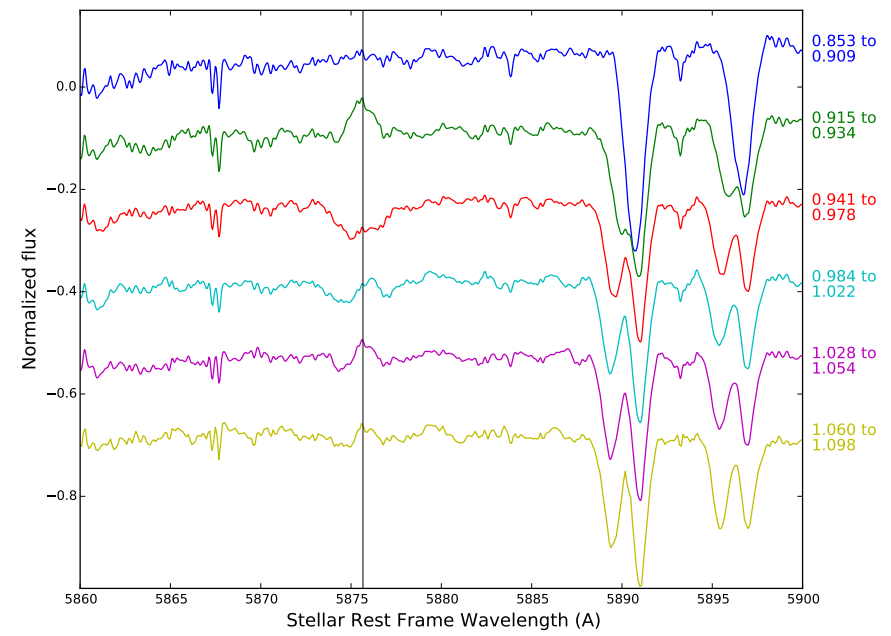

Fig. 3. Evolution of the He I $\lambda 5875.66$ (D3) line profile of RR Lyr. D3 appears in emission at $\varphi=0.909$ and is clearly visible until $\varphi=0.928$. A weak post-maximum emission is also observed between phases 1.028 and 1.054 .

is first weakly blueshifted $\left(-4 \mathrm{~km} \mathrm{~s}^{-1}\right)$ before reaching a maximum value of $14 \mathrm{~km} \mathrm{~s}^{-1}$ at $\varphi=0.922-0.928$. In parallel, the $\mathrm{H} \alpha$ emission component is much more blueshifted, $-90 \mathrm{~km} \mathrm{~s}^{-1}$ at $\varphi=0.903$ and the decrease to $-33 \mathrm{~km} \mathrm{~s}^{-1}$ at $\varphi=0.941$. The maximum intensity of the He I $\lambda 5875.66$ emission is near $5 \%$ of the continuum at $\varphi=0.922-0.928$ and occurs at the same time as the maximum $\mathrm{H} \alpha$ emission (being $I=35 \%$ of the continuum).

It seems (see Fig. 3) that a Schwarzschild's mechanism i.e., a line-doubling phenomenon, follows the emission, but the phase when the blueshifted absorption component is weaker than the redshifted one is not visible. This is certainly the consequence of too low a $\mathrm{S} / \mathrm{N}$ for our data.

As reported by Preston (2011) and Gillet et al. (2013), we also observed the reapparance of a weak D3 emission after maximum light. It seems to appear near phase 1.028 until approximately $\varphi=1.054$ (see Fig. 4) and is just above the continuum 


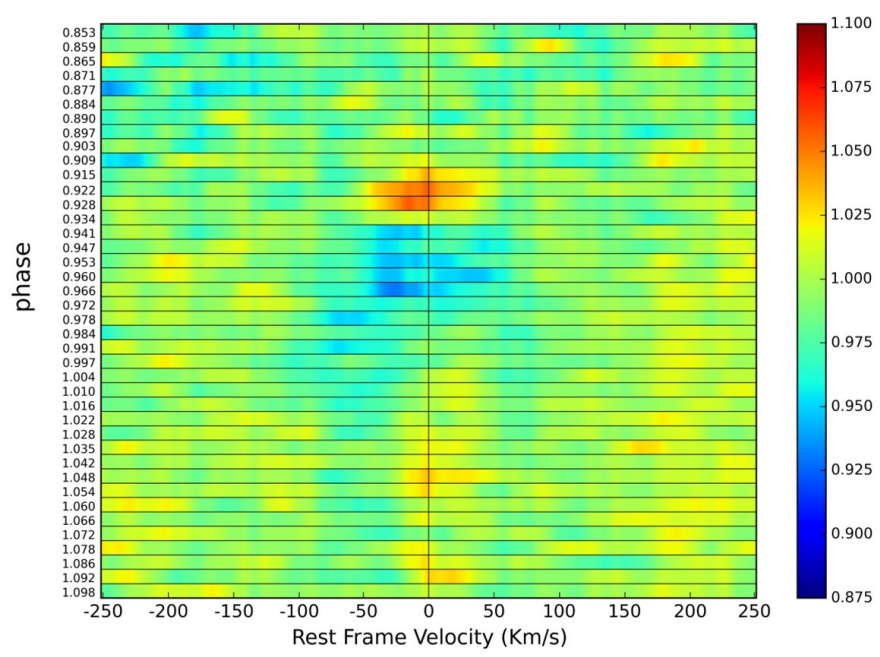

Fig. 4. Time series of the He I $\lambda 5875.66$ (D3) line of RR Lyr. D3 appears in emission at $\varphi=0.909$ and is clearly visible until $\varphi=0.928$. A weak post-maximum D3 emission is also observed between phases 1.028 and 1.054 .

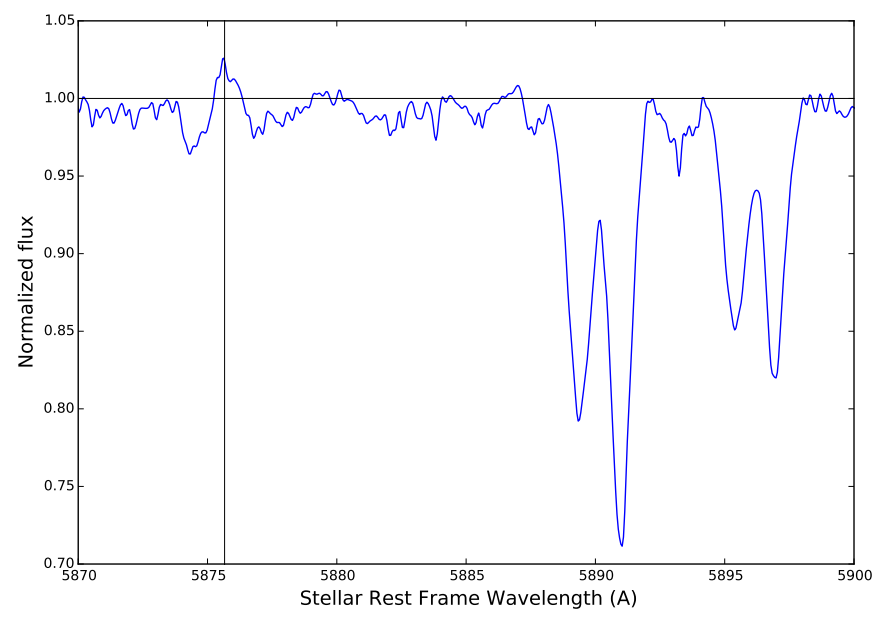

Fig. 5. Profile of He I $\lambda 5875.66$ line (D3) in the spectrum of RR Lyr obtained by co-adding five spectra obtained in the phases: 1.028, 1.035, $1.042,1.048$, and 1.054. The position of D3 is marked by a vertical line and the approximative level of the continuum by a horizontal line.

( $2 \%$ ). During this second He I emission, the $\mathrm{H} \alpha$ line presents a single blueshifted absorption profile (Fig. 2). Preston (2011) co-added eighteen spectra of RV Oct obtained in the phase interval $\varphi=1.00-1.06$ to obtain the spectrum displayed in his Fig. 3, which is very similar to our co-adding five spectra obtained in the phase interval 1.03-1.05 (Fig. 5).

The second helium line for which we have also detected emission is He I 16678.16 (singlet). It appears in emission on two successive spectra at $\varphi=0.922$ and $\varphi=0.928$ (Fig. 6) i.e., when both $\mathrm{H} \alpha$ and $\mathrm{D} 3$ show their maximum emission. The maximum intensity of He I $\lambda 6678.16$ is around $1 \%$ above the contunuum at $\varphi=0.928$. However, unlike D3, no post-maximum emission is observed (Fig. 6). A careful analysis of our observations shows that no He II emission is detected around phases $\varphi=0.922$ and $\varphi=0.928$.

\section{Physical origin of the D3 helium P-Cygni profile}

Recently, Gillet (2014) has suggested that the $\mathrm{H} \alpha$ profile of longperiod Cepheids turns into a P-Cygni type profile when the shock

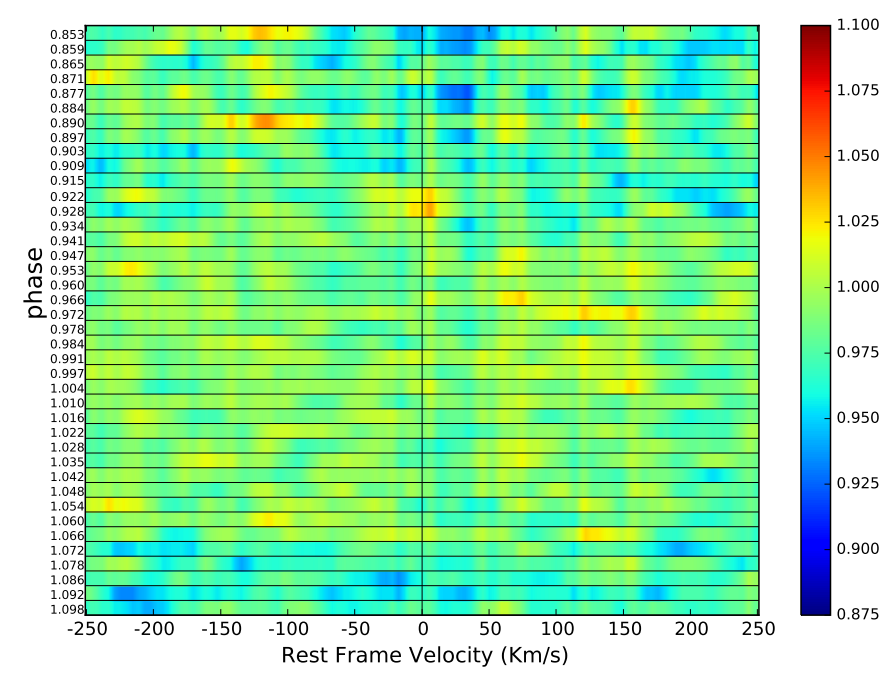

Fig. 6. Time series of the He I $\lambda 6678.16$ line of RR Lyr. Weak emission on two successive spectra at $\varphi=0.922$ and $\varphi=0.928$ is present i.e., when $\mathrm{H} \alpha$ and $\mathrm{D} 3$ show their maximum emission intensity. There is not post-maximum emission unlike the D3 line.

wave reaches the high atmosphere. More precisely, when the shock is emerging from the photosphere, its receding part is occulted by the stellar disk, and consequently, only the advancing part of the shock, hence blueshifted, can be observed. Then, due to its propagation through the atmosphere, the shock ends up detached from the photosphere. The receding part of the shock thus becomes more and more visible and produces a redshifted emission component. Finally, because the shock is detached from the photosphere, the blueshifted emission component gradually disappears, and a P Cygni profile is formed.

Gillet \& Fokin (2014) suggested that the weak redshifted emission component occurring around the pulsation phase $\varphi=$ 0.30 in RR Lyr can be also interpreted as a P-Cygni profile. The shock propagation toward the high atmosphere of the star could be the cause of this progressive and striking line profile change of the line profile. Of course, depending on the shock intensity, the redshifted emission component can be observed over or under the continuum.

If we assume that the D3 helium near the pulsation phase $\varphi=1.04$ is built as a P-Cygni profile, then following Gillet $\&$ Fokin (2014), the shock front velocity is around $60 \mathrm{~km} \mathrm{~s}^{-1}$. From the $\mathrm{H} \alpha$ profile Gillet \& Fokin (2014) found that the shock velocity varies from $130 \mathrm{~km} \mathrm{~s}^{-1}$ at $\varphi=0.902$ to $115 \mathrm{~km} \mathrm{~s}^{-1}$ at $\varphi=0.927$. This means that the shock strongly decelerates between the pulsation phases 0.902 and 1.04. In fact, the shock velocity decreases by more than half its initial value in only $15 \%$ of the pulsation cycle.

The "third apparition" of $\mathrm{H} \alpha$ emission (Preston 2011) has not yet been observed in RR Lyr itself. This weak post-maximum redshifted emission has been observed in the red absorption wing of the $\mathrm{H} \alpha$ profile in RV Oct, WY Ant, and V1645 Sgr (Chadid \& Preston 2013). It appears during declining light and reaches its maximum strength near phase $\varphi=0.30$. According to Gillet \& Fokin (2014), this corresponds to an average shock velocity near $80 \mathrm{~km} \mathrm{~s}^{-1}$. If this velocity also applies to RR Lyr, then after a deceleration phase, the shock should endure a moderate acceleration phase in the upper atmosphere

When the shock becomes visible through emission lines, it emerges from the deep photosphere. At this stage, emissions of hydrogen and helium lines are the most intense. Then, when the 
shock is crossing the above atmospheric layers, it first undergoes a significant deceleration owing to large energy loss by radiation (Fadeyev \& Gillet 2001). After this phase, the shock propagates away from the photosphere, i.e., after the appearance of the P-Cygni profile of the D3 line, its intensity increases enough to produce weak emission of the D3 helium line. Both the substantial width of the $\mathrm{H} \alpha$ profile and the line-doubling phenomenon obviously do not allow to observe an emission within the $\mathrm{H} \alpha$ line profile near the pulsation phase 1.04. This, the $\mathrm{H} \alpha$ emission should appear later, around phase 0.30 , if the shock intensity is strong enough at this phase. Additional observations are thus required to confirm the presence or not of this emission phase.

The presence of a D3 P-Cygni profile implies that the amplitude of the ballistic motion of the extended atmosphere is exceptionally wide. If a P-Cygni profile is also observed for the $\mathrm{H} \alpha$ line, then the ballistic movement has even larger amplitude, especially if the shock velocity increases. Finally, the observation of P-Cygni emissions allows estimation of the associated shock velocities that govern the dynamics of the atmospheric layers, especially within the upper atmosphere.

\section{Conclusion}

For the first time, we have reported observing the post-maximum emission of the He I 25875.66 (D3) line in RR Lyr itself, the prototype of the RR Lyrae stars. This weak emission has the form of a P-Cygni profile, with the maximum of the emission centered at the zero velocity in the stellar restframe. We suggested that this profile is a natural consequence of the large extension of the expanding atmosphere induced by the main strong shock occurring at each pulsation cycle. This weak helium emission occurs at the end of the $\mathrm{H} \alpha$ line-doubling phenomenon, the large width of this latter certainly preventing similar weak emission. Also, it might be that around the
$\mathrm{H} \alpha$ line-forming region, the shock is still not strong enough to produce any appreciable emission. The helium P-Cygni profile occurs after the maximum light near $\varphi=1.04$. We infer that the shock velocity at this phase is around $60 \mathrm{~km} \mathrm{~s}^{-1}$. This suggests that the shock wave suffered a sharp deceleration between its photosphere emergence and its its detachment off the photosphere because the initial shock velocity is estimated to be close to $130 \mathrm{~km} \mathrm{~s}^{-1}$. After the appearance of the D3 emission, we expect that the shock velocity increases, but further observations are required to confirm this hypothesis.

Acknowledgements. Spectroscopic data were reduced with the part dedicated to the spectroscopy of the free and open source astronomy software AudeLA (http://www.audela.org/) written by Benjamin Mauclaire (bma.ova@gmail.com).

\section{References}

Chadid, M., \& Gillet, D. 1996a, A\&A, 308, 481

Chadid, M.,\& Preston, G. W. 2013, MNRAS, 434, 552

Fadeyev, Yu. A.,\& Gillet, D. 2001, A\&A, 368, 901

Fokin, A. B., \& Gillet, D. 1997, A\&A, 325, 1013

Gillet, D. 2014, A\&A, 568, A72

Gillet, D., \& Crowe, R. A. 1988, A\&A, 199, 242

Gillet, D., \& Fokin, A. B., 2014, A\&A, 565, A73

Gillet, D., Fabas, N., \& Lèbre, A. 2013, A\&A, 553, A59

Kolenberg, K., Fossati, L., Shulyak, D., et al. 2011, A\&A, 519, A64

Le Borgne, J.-F., Poretti, E., Klotz, A., 2014, MNRAS, 441, 1435

Preston, G. W. 2009, A\&A, 507, 1621

Preston, G. W. 2011, AJ, 141, 6

Schwarzschild, M., 1952, Transaction of the IAU VIII, ed. P. Th. Oosterhoff (Cambridge University Press), 811

Thizy, O., \& Cochard, F. 2010, in Active OB stars: structure, evolution, mass loss, and critical limits, eds. C. Neiner, G. Wade, G. Meynet, \& G. Peters, Proc. IAU Symp., 272, 282

Wallerstein, G., 1959, ApJ, 130, 560

Whitney, C., 1956, Ann. Astrophys., 19, 34 